|| Print ISSN: 2589-7837 || Online ISSN: 2581-3935 ||

International Journal of Medical Science and Diagnosis Research (IJMSDR)

Available Online at www.ijmsdr.com

NLM (National Library of Medicine ID: 101738824)

Original Research Article

Volume 6, Issue 1; January: 2022; Page No. 01-08

\title{
Pseudoexfoliation Glaucoma and its Systemic Association: A Clinical Study
}

\author{
Dr Lakshmi B Ramamurthy ${ }^{1}$, Dr Sahana Nemmar Chandrashekarabhatta ${ }^{2}$, \\ Dr Sudheendra B $\mathbf{R}^{3}$ \\ ${ }^{1}$ Assistant Professor, Dept of Ophthalmology, Karnataka Institute of Medical Sciences, \\ HUBLI \\ ${ }^{2}$ Junior Resident, Dept of Dermatology, Bangalore Medical College and Research Institute, \\ Bengaluru \\ ${ }^{3}$ Junior resident, Dept of General Medicine, Bangalore Medical College and Research \\ Institute, Bengaluru
}

Conflicts of Interest: Nil

Corresponding author: Dr Sahana Nemmar Chandrashekarbhatta

DOI: https://doi.org/10.32553/ijmsdr.v6i1.891

\begin{abstract}
:
Pseudoexfoliation [PXF] being a age related elastotic process is a well known entity among all ophthalmologists. PXF is most common form of secondary glaucoma.

Objective: To study the clinical presentations and response to treatment of PXF glaucoma and also to throw light on its systemic associations.

Methods: A total of 70 eyes of PXF glaucoma was considered for the study in duration of 6 months from june to November 2021 at a tertiary care center. All patients underwent detailed ophthalmic evaluation along with gonioscopy, optic disc assessment, visual fields and intraocular pressure. Based on all these, patients were graded as mild, moderate and advanced glaucoma and treated accordingly either with medical or surgical line of management. Patients were followed up for a duration of 6 months. ECG, echocardiography and dermatological evaluation of all the patients were done.
\end{abstract}

Results: Mean age of 70 patients was 52.2 years and there was male preponderance .Powdery greyish white pseudoexfoliative material on pupillary margin was seen in 41 eyes[58.5\%] and on anterior capsule was seen in 19 eyes[27.1\%]. IOP in these 70 patients ranged between 14 to 40mmHg. Gonioscopy showed exfoliative material deposition in 14 eyes[20\%] and increased pigmentation of trabecular meshwork in 44 eyes[62.85\%]. Also, it revealed narrow angle( grade $1 \& 2$ ) in 6 eyes and open angle (grade $3 \& 4$ ) in rest .Visual filed changes were present in 57 of 70 eyes with PXF glaucoma. Optic disc changes in PXF glaucoma also showed variations with 17 eyes having CD ratio of $<0.5$, while 38 eyes had a cupping between $0.6-0.8$. Eleven eyes showed advanced cupping of $>0.8$ while 4 eyes had glaucomatous optic atrophy. 60 eyes were treated with medical line while 10 eyes were managed surgically. 4 patients had dermal nodules and diastolic dysfunction was noted in 31 patients.

Conclusion: PXF confers a significantly higher risk of developing glaucoma in comparison with the general population and needs close monitering. PXF glaucoma and its systemic associations also has to be considered

Keywords: PXF, pseudoexfoliative, ECG 


\section{Introduction:}

Pseudoexfoliation is an age related degenerative process, with deposition of greyish white fibrillar material in various ocular structures like lens, iris, trabecular meshwork and so on ${ }^{1}$. Pseudoexfoliation (PXF) syndrome is not only an ocular disease but a generalized disorder involving abnormal production or turnover of extracellular matrix material or both ${ }^{2}$ Pseudoexfoliation syndrome (PXF) was first reported by Lindberg in 1917 in a Finnish population ${ }^{3}$. Prevalence of pseudoexfoliation occurs world-wide, however, there are geographic differences in prevalence rates with highest rates in Scandinavian countries ${ }^{4}$ Considering the Indian scenario, the prevalence of PXF based on hospital reports from India varies between $1.87 \%$ and $13.5 \%{ }^{5}$. The prevalence of patients with PXF in South India is $0.6 \%$ and increase in the prevalence of PXF was noted with increasing age. ${ }^{6}$

PXF syndrome which was initially termed as "glaucoma capsulare" is associated with increased risk of glaucoma. This is associated with deposition of flakes of PXF material in angle with increased pigmentation in angle termed as Sampaolesi's line. PXF glaucoma is also associated with narrow angles secondary to zonular weakness .7 Pseudoexfoliative glaucoma increases with age and has a higher prevalence in patients between $60-70$ years ${ }^{8}$. Glaucoma is most frequent association with PXF and hence both forms of open angle and angle closure are associated with PXF syndrome.

The electron microscopic demonstration of PXF material in various visceral organs was given by Schlotzer-Schrehardt ${ }^{9}$. Hence we are dealing with a generalized process and not with a peculiar ocular phenomenon. There is definite involvement not only of the skin but of the heart, lung, liver, kidney, cerebral meninges, and gall bladder. ${ }^{10}$ Also cardiac involvement in the form of diastolic dysfunction was noted in eyes with pseudoexfoliative glaucoma ${ }^{11}$.

With this background, we wanted to assess and analyze PXF glaucoma and its clinical correlates.

\section{Methodology:}

A hospital based prospective study was done at a tertiary care center during the study duration of 6 months from June to November 2021 . Patients belonging to either sex diagnosed to have pseudoexfoliation based on slit lamp examination and having glaucoma were included in the study. Patients with other causes of glaucoma like steroid induced, traumatic, inflammatory and other secondary causes of glaucoma were excluded.

Patients fulfilling the inclusion criteria were recruited into the study after obtaining the informed consent from the participants. Study adhered to guidelines of declaration of Helsinki. Ophthalmological workup was done in all patients which included-Visual acuity testing for near and distant vision, slit lamp biomicroscopy for evidence of exfoliation material in anterior segment, before and after dilatation of pupil, examination of cornea, anterior chamber depth, iris, pupil and lens were made .

Initial ophthalmological workup was followed by refraction and correction (if required),tonometry using applanation tonometry, gonioscopy. fundoscopy ,visual fields testing.

Patients with pseudoexfoliative glaucoma were classified into mild, moderate, severe and advanced based on parameters- IOP, angle configuration, cupping and visual field defects. 


\begin{tabular}{|l|l|l|l|}
\hline \multicolumn{4}{|c|}{ Table 1: Criteria for glaucoma grading in the study } \\
\hline GRADE & CUPPING & $\begin{array}{l}\text { Visual } \\
\text { Changes }\end{array}$ & $\begin{array}{l}\text { Intraocular } \\
\text { Pressure }\end{array}$ \\
\hline MILD & $<0.5$ & Minimal & $14-18$ \\
\hline MODERATE & $0.5-0.7$ & Arcuate Defects & $16-22$ \\
\hline SEVERE & $>0.8$ & Advanced defects & $>22$ \\
\hline ADVANCED & GOA & Advanced defects & HIGH \\
\hline
\end{tabular}

Gonioscopic record of angle was graded based on Shaffer's grading:

\begin{tabular}{|c|l|l|}
\hline \multicolumn{3}{|c|}{ Table 2: Shaffer's gonioscopic grading of angle } \\
\hline Grade 4 & $\left(35-45^{\circ}\right)$ & Ciliary body can be visualized. \\
\hline Grade 3 & $\left(25-35^{\circ}\right)$ & Open angle in which at least the scleral spur can be identified. \\
\hline Grade 2 & $\left(20^{\circ}\right)$ & $\begin{array}{l}\text { Moderately narrow angle in which only the trabeculum can be } \\
\text { identified. }\end{array}$ \\
\hline Grade 1 & $\left(10^{\circ}\right)$ & $\begin{array}{l}\text { Very narrow angle in which only Schwalbe line, and perhaps also the } \\
\text { top of the trabeculum, can be identified. }\end{array}$ \\
\hline $\begin{array}{c}\text { Slit } \\
\text { angle }\end{array}$ & & $\begin{array}{l}\text { No obvious iridocorneal contact but no angle } \\
\text { identified. }\end{array}$ \\
\hline Grade 0 & $\left(0^{\circ}\right)$ & Closed angle due to iridocorneal contact \\
\hline $\begin{array}{l}\text { Adapted from: } \\
\text { approach. Uveitis, } \text {. Sth edition: Elsevier, 2015:397-399. }\end{array}$ \\
\hline
\end{tabular}

Patients with mild glaucoma were prescribed with single topical drug .Patients with moderate glaucoma were treated by combination of two drugs. Any evidence of progression in the above groups were considered for trabaculectomy. Patients with severe and advanced glaucoma underwent surgical line of management for glaucoma in the form of trabaculectomy and also combined procedure with cataract surgery in required caeses. Patients with absolute glaucoma were considered for cyclocryotherapy. Patients in all the above mentioned categories were followed up for a duration of 6 months and treatment protocol was assessed. Gonioscopy, fundoscopy and tonometry was done in each visit at intervals of 2 months up to 6months.

All patients were also subjected to ECG and echocardiographyto assess cardiac function and also dermatology opinion was taken regarding skin changes if any. Descriptive statistics has been applied to analyze the collected data and results were analysed accordingly. Observations are tabulated and represented as percentages and mean values in appropriate places.

\section{Results:}

A total of 70 eyes were considered in the study. Mean age was 52.2 years and there was male preponderance .Powdery greyish white pseudoexfoliative material on pupillary margin was seen in 41 eyes[58.5\%] and on anterior capsule was seen in 19 eyes[27.1\%]. IOP in these 70 patients ranged between 14 to $40 \mathrm{mmHg}$ with 26 of them having IOP between $19-26 \mathrm{~mm} \mathrm{hg}$. IOP ranges in these eyes are mentioned in table 3 . 


\begin{tabular}{|l|l|l|}
\hline \multicolumn{3}{|c|}{ Table 3: Intraocular pressure in PXF-Glaucoma eyes } \\
\hline Intraocular pressure & Number of eyes & Percentage \\
\hline$<16$ & 19 & 27.14 \\
\hline $17-21$ & 26 & 37.14 \\
\hline $21-30$ & 9 & 12.85 \\
\hline $31-40$ & 13 & 18.57 \\
\hline$>41$ & 3 & 4.28 \\
\hline Total & 70 & 100 \\
\hline
\end{tabular}

Gonioscopy showed exfoliative material deposition in 14 eyes[20\%] and increased pigmentation of trabecular meshwork in 44eyes[62.85\%]. Also, it revealed grade 1 angle in 3 eyes, grade 2 in 3 eyes, grade 3 in 31 eyes and grade 4 in 33 eyes.Visual filed changes were present in 57 of 70 eyes with PXF glaucoma. Optic disc changes in PXF glaucoma also showed variations with 17 eyes having $C D$ ratio of $<0.5$, while 38 eyes had a cupping between 0.6-0.8. Eleven eyes showed advanced cupping of $>0.8$ while 4 eyes had glaucomatous optic atrophy. Graph 1 shows the variations in optic disc changes. Based on combination of above mentioned parameters, PXFglaucoma was graded and has been represented in table-4

\begin{tabular}{|l|l|l|}
\hline \multicolumn{3}{|c|}{ Table 4: Glaucoma grading in PXF eyes } \\
\hline GRADE & No of eyes & Percentage \\
\hline MILD & 19 & 27.14 \\
\hline MODERATE & 26 & 37.14 \\
\hline SEVERE & 6 & 8.57 \\
\hline ADVANCED & 16 & 22.85 \\
\hline ABSOLUTE & 3 & 4.28 \\
\hline & 70 & 100 \\
\hline
\end{tabular}

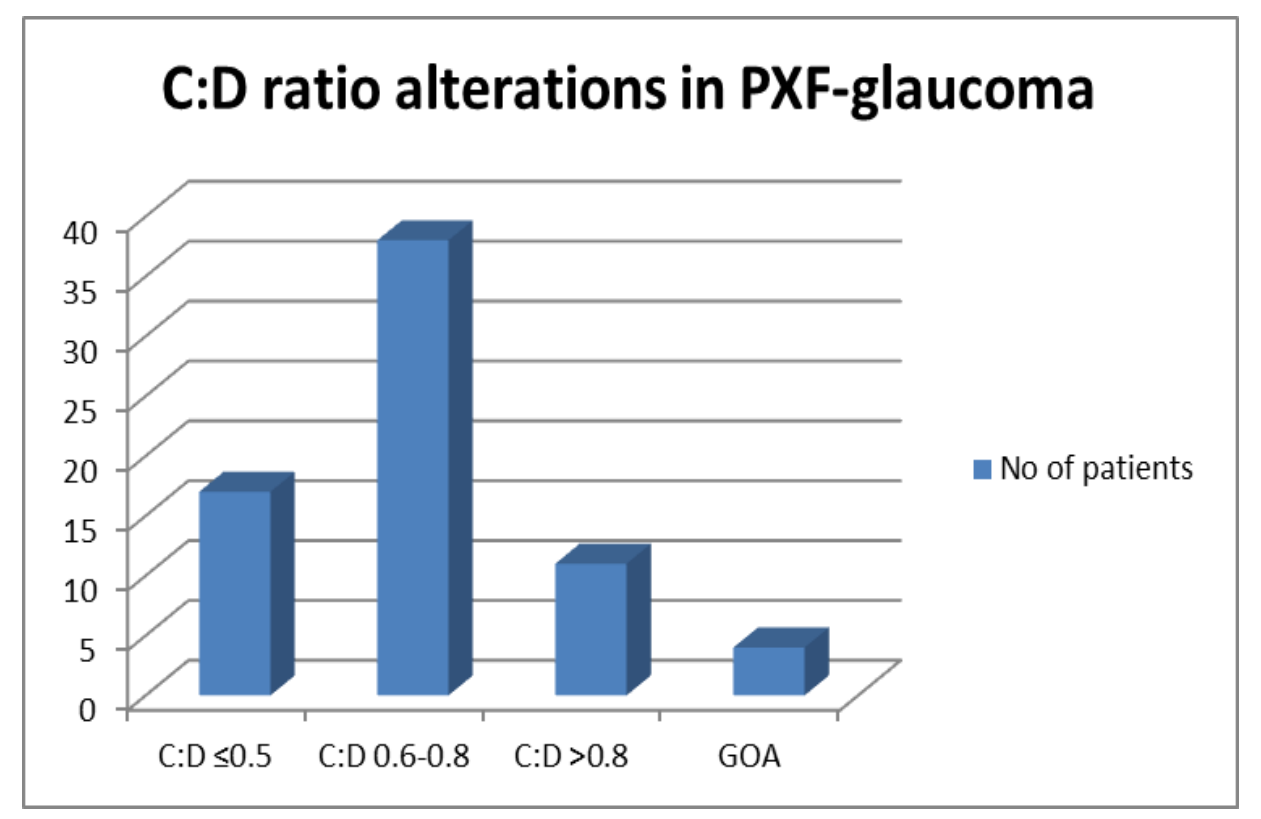




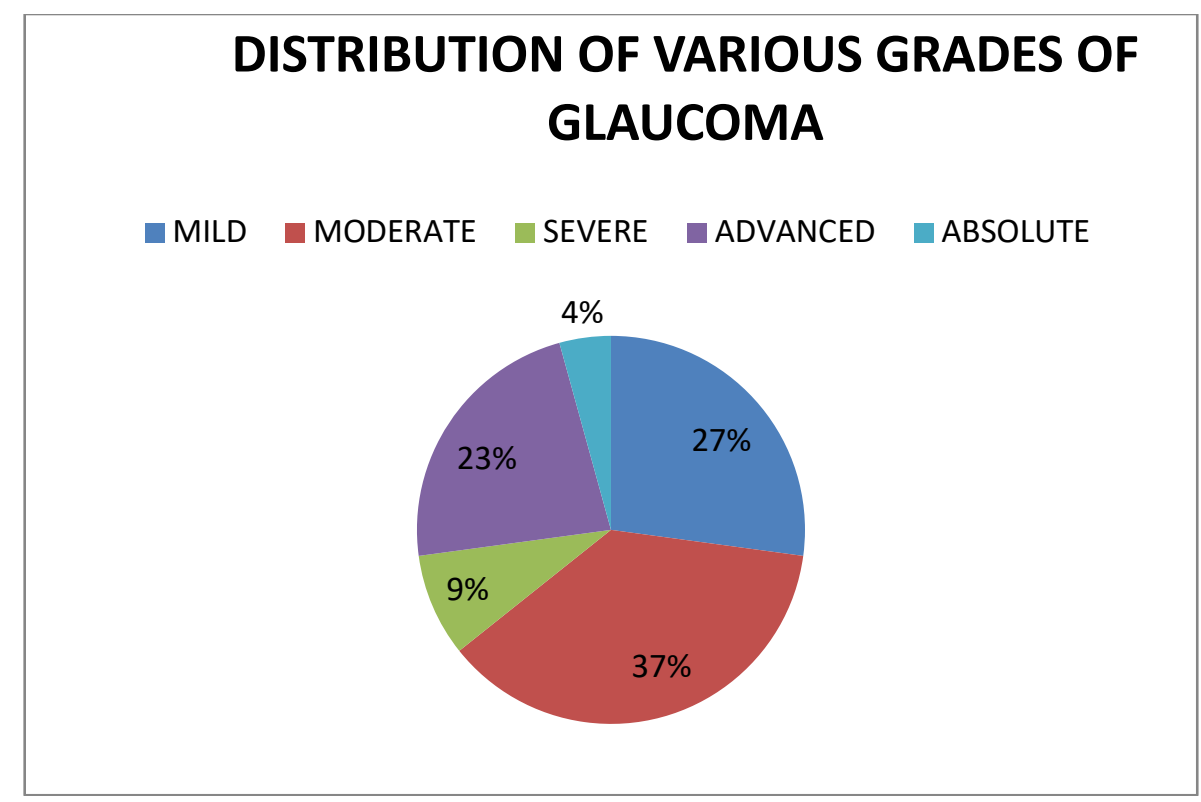

Based on grading, PXF- glaucoma was managed by medical, surgical or combined treatment. Medical line included topical anti glaucoma medications either single drug, dual or triple drug regimen, while surgical line included trabaculectomy or trabaculectomy combined with cataract surgery. 60 eyes were managed with medical line of management with 19 on single drug , 26 on dual drug and 15 $\mathrm{n}$ triple drug regimen respectively. 10 eyes were managed surgically while 6 of them needed additional on drug for control of IOP postoperatively.

All patients with mild glaucoma - were managed on one drug. During the follow up over 6 months, only 2 patients required addition of another drug in due course of follow-up. These patients who progressed had $\mathrm{C}$ : D ratio 0.6 with visual field defects. All patients with moderate glaucoma were managed with two anti-glaucoma medications, and 4 of them showed progression over follow up period. These patients had 0.7 cupping and arcuate field defects. One patient also had narrow angle for which peripheral iridotomy was done. Four patients had bilateral moderate glaucoma , 3 patients had moderate glaucoma in one eye and mild glaucoma in other eye and
1 patient had moderate glaucoma in one eye and advanced glaucoma in other eye.

Severe glaucoma was treated with either medical or surgical line of management. Trabaculectomy was done in 2 patients with severe glaucoma who remained stable in follow-up period. Rest were treated with either 2 or 3 anti-glaucoma topical medications.

Out of thirteen patients with advanced glaucoma, 8 patients underwent trabaculectomy. Two patients refused surgery and rest 3 patients were managed with combination on beta blocker with alpha agonist and prostaglandins. In the follow-up period, 2 patients had well controlled IOP without signs of progression, whereas 6 patients required topical beta blocker even after trabaculectomy for maintaining IOP at target level

In the course of follow-up of PXF-glaucoma, any signs of increase in baseline intraocular pressure [uncontrolled IOP], increase in cupdisc ratio and advancing visual field defects were considered as signs of glaucoma progression. A total of eleven eyes showed progression in the follow-up period out of which 2 eyes were in mild glaucoma group , 3 with moderate glaucoma and 6 in advanced glaucoma. 
Also we noted that there was decreased ejection fraction of less than $40 \%$ in 31 patients( $44.3 \%)$ with 4 patients having history of ischemic heart disease. Dermatological evaluation revealed increased skin laxity in 47 patients $(67 \%)$ with 4 of them showing nodular skin changes.

\section{Discussion:}

Pseudoexfoliation (PXF) is an age-related disorder characterized by the accumulation of a fibrillar extracellular material in ocular tissues and is often associated with glaucoma. Its morphological manifestation includes accumulation of fibrillar material deposits in ocular tissues which may be a ground for various ocular pathologies, in particular the open-angle glaucoma for which the syndrome is the most common identifiable cause. ${ }^{13}$ In patients with ocular deposits aggregates of similar material were recently identified in the skin as well as in some internal organs e.g. heart, lung, liver, kidney.

Genetic basis currently describes LOXL1 as the most significant genetic risk factor for glaucoma in general. LOXL1 is a key enzyme involved in elastic fibre synthesis and homeostasis supporting a role of elastogenesis and elastosis in the pathophysiology of PXF syndrome ${ }^{14}$ A specific association between PXF and elastic elements suggests it to be a type of elastosis found in skin and conjunctival biopsy $^{9}$. In the myocardium the pseudoexfoliation materials were closely apposed to the myocardial cells and their interrupted basement membranes ${ }^{11}$. A possible asymptomatic diastolic left ventricular dysfunction without overt failure was tested as hypothesis suggests suggests the possibility of an association between patients with PXF syndrome and a discrete asymptomatic myocardial diastolic dysfunction ${ }^{15}$.

In our study, out of 70 eyes, high IOP was noted in 49 eyes and 44 eyes had gonioscopic evidence of fibrillary PXF material pointing towards definitive diagnosis of PXF-induced glaucoma. All of them had grade 3-4 angle except in 6 patients with angle grading was between 1-2 (suggestive of closed angle). A similar study on pseudoexfoliative glaucoma, shows $66 \%$ having exfoliative material at pupillary margin ${ }^{16}$ comparable to $58 \%$ in our study. A study from northern china shows increased pigmentation in $49.3 \%$ and flecks of PXF as $9.3 \%$ were seen on the trabecular meshwork, comparable to $60 \%$ and $20 \%$ in our study ${ }^{16,17}$.This finding is not exclusive to PXF, but can be seen in pigment dispersion syndrome and chronic inflammation. ${ }^{17}$ Increased pigmentation in the angle has been correlated with increased IOP. ${ }^{18,19}$ This suggests that pigmentation might provide a clinical gauge as to the severity of glaucomatous disease. Patients with PXF who were untreated progressed more quickly than treated individuals. The risk of progression was calculated to be 2-fold higher in individuals with $\mathrm{PXF}^{4}$. Hence 11 eyes in our study also showed progression supporting that, PXF glaucoma has potential of rapid progression.

Dermatological changes owing to elastosis in PXF represents its systemic involvement. Found in upto $80 \%$ of patients. ${ }^{20}$ Dermatologist evaluation showed 47 of them having elstotic changes, but owing to aging related process ${ }^{20}$ , its true association is questionable. However 4 patients had nodular skin changes suggestive of systemic involvement . A skin biopsy with immunohistology would have helped in further correlation, remains drawback of the study. A study by Bojic et al conducted to investigate the frequency of asymptomatic left ventricular dysfunction in patients with pseudoexfoliation syndrome suggests the possibility of its association with discrete asymptomatic myocardial diastolic dysfunction. We observed that there was diastolic dysfunction on 2D echo in 31 patients, but the confounders like increasing age itself a risk factor also has to be kept in mind. A better systemic evaluation considering hypertensive status and other 
detailed evaluation would have helped to correlate this association more strongly.

\section{Conclusion:}

In association with the higher prevalence of ocular hypertension and/or glaucoma, PXF confers a significantly higher risk of developing glaucoma in comparison with the general population. PXF glaucoma has to be diagnosed and treated promptly without any delay to save patients' vision. Based on recent evidence, an eye has to be kept on its systemic associations too. Physician, dermatological and other multispeciality approach helps to manage the patients comprehensively for a better outcome.

\section{References:}

1. Ritch R, Schlötzer-Schrehardt U. Exfoliation syndrome. Surv Ophthalmol. 2001;45:265-315. [PubMed]

2. Naumann GO, Schlötzer-Schrehardt U, Küchle M. Pseudoexfoliation syndrome for the comprehensive ophthalmologist: intraocular and systemic manifestations. Ophthalmology. 1998 Jun 1;105(6):95168.

3. Lindberg JG: Kliniska undersokningar over depigmenteringen av pupillarranden och genomlysbarheten av iris vid fall av aldersstarr samt i normala ogon hos gamla personer. [Clinical studies of depigmentation of the pupillary margin and transillumination of the iris in cases of senile cataract and also in normal eyes in the aged] [Thesis]. 1917, Helsinki, Finland: Helsinki University.

4. Desai MA, Lee RK. The medical and surgical management of pseudoexfoliation glaucoma. International ophthalmology clinics. 2008;48(4):95.

5. Thomas R, Paul P, Muliyil J. Glaucoma in India. J Glaucoma.2003;12:81-7.

6. Vijayalakshmi V, Desai S, Prasanna N, Sreelakshmi G. Pseudoexfoliation Syndrome: Prevalence In South Indian Population. International Journal Of
Medical Science And Clinical Inventions Volume 2 issue 032015 page no. 766-771 ISSN: 2348-991X

7. Thomas R, Paul P, Muliyil J. Glaucoma in India. J Glaucoma.2003;12:81-7.

8. Plateroti P, Plateroti AM, Abdolrahimzadeh S, Scuderi G. Pseudoexfoliation syndrome and pseudoexfoliation glaucoma: a review of the literature with updates on surgical management. Journal of ophthalmology. 2015 Oct 29;2015.

9. Schliitzer-Schrehardt UM, Koca MR, Naumann GO, Volkholz H. Pseudoexfoliation syndrome. Ocular manifestation of a systemic disorder? Arch Ophthalmol 1992; 110: 1752-6.

10. 10. Gottfried O.H Naumann; Ursula Schlötzer-Schrehardt; Michael Küchle (1998). Pseudoexfoliation syndrome for the comprehensive ophthalmologist: Intraocular and systemic manifestations. , 105(6), 0-968. doi:10.1016/s01616420(98)96020

11. Bojić L, Ermacora R, Ivanisević $M$, Galetović D, Mandić Z, Novak-Laus K, Cerovski B. Doppler-echocardiographic characteristics of left ventricular function in patients with pseudoexfoliation glaucoma: a preliminary report. Coll Antropol. 2005;29 Suppl 1:5-8. PMID: 16193666.

12. Jack J Kanski, Brad Bowling. Clinical Ophthalmology: a systematic approach. Uveitis, 8th edition: Elsevier, 2015:397399.

13. Pathogenesis and histopathology of pseudoexfoliative lesions. The eyeball disease or ocular manifestation of a generalized process?]. Przegl Lek. 2006;63(7):588-92. Polish. PMID: 17203815.

14. Wiggs JL. Association between LOXL1 and pseudoexfoliation. Arch Ophthalmol. 2008;126:420-1. 
15. Pseudoexfoliation syndrome and asymptomatic myocardial dysfunction. Graefes Arch Clin Exp Ophthalmol. 2005 May;243(5):446-9. doi: 10.1007/s00417004-1074-9. Epub 2004 Dec 14. PMID: 15599584.

16. Ren R, Ding J, Wang N, Teng CC, de Moraes GV, Jonas JB, Ritch R. Clinical Signs and Characteristics of Exfoliation Syndrome and Exfoliative Glaucoma in Northern China. The Asia-Pacific Journal of Ophthalmology. 2015 Mar 1;4(2):86-8.

17. Gillies W. Pseudoexfoliation of the lens capsule and pigmentary glaucoma. Trans Ophthalmol Soc Austral 1962:22.

18. Puska P. The amount of lens exfoliation 21. exfoliation syndrome with or without glaucoma. Acta Ophthalmol Scand 1995;73:226-232. [PubMed: 7493233]

19. Shuba L, Nicolela MT, Rafuse PE. Correlation of capsular pseudoexfoliation material and iridocorneal angle pigment with the severity of pseudoexfoliation glaucoma. J Glaucoma 2007;16:94-97.

20. Streeten BW, Dark AJ, Wallace RN, Li ZY, Hoepner JA. Pseudoexfoliative fibrillopathy in the skin of patients with ocular pseudoexfoliation. Am J Ophthalmol. 1990 Nov 15;110(5):490-9. doi: $\quad 10.1016 / \mathrm{s} 0002-9394(14) 77871-7$. PMID: 2240134. 